\title{
BASELINE OF VIRTUAL CAMERAS ACQUIRED BY A STANDARD PLENOPTIC CAMERA SETUP
}

\author{
Christopher Hahne, Amar Aggoun, Shyqyri Haxha, Vladan Velisavljevic, and Juan C. J. Fernández
}

\author{
Dept. of Computer Science, University of Bedfordshire
}

Park Square, Luton, Bedfordshire, LU1 3JU, United Kingdom

\begin{abstract}
Plenoptic cameras have been used to computationally generate viewpoint images from the captured light field. This paper aims to provide a prediction of corresponding virtual camera positions based on the parameters of a standard plenoptic camera setup. Furthermore, by tracing light rays from the sensor to the object space, a solution is proposed to estimate the baseline of viewpoints. By considering geometrical optics, the suggested approach has been implemented in Matlab and assessed using Zemax, a real ray tracing simulation tool. Thereby, the impact of different main lens locations is investigated. Results of the baseline approximation indicate that estimates obtained by the proposed model deviate by less than $0.2 \%$ compared to the complex real ray tracing method.
\end{abstract}

Index Terms - Baseline, estimation, plenoptic camera, ray tracing, virtual camera, multiview, autostereoscopy

\section{INTRODUCTION}

With Integral Imaging (II), it has always been the major objective to capture and display Three-Dimensional (3-D) content with the aid of an array of optical elements. Early research in the field of II was conducted by Lippmann [1] in 1908 by using a Micro Lens Array (MLA) to acquire 3-D image data. In the more recent past, autostereoscopic displays as well as plenoptic cameras [2] have been designed. A large and growing body of literature [3, 4] has investigated ray tracing in plenoptic cameras starting from the object space with the aid of similar triangles. An attempt to examine the position of virtual lenses in focused plenoptic cameras has been developed by Georgiev et al. [4]. In this work, virtual lens positions are provided by using the concept of principal planes in a lens system consisting of two lenses, namely a micro lens and a main lens. For the time being, that approach does not succeed in estimating the baseline of virtual cameras which is necessary to screen plenoptic 3-D content on multiview displays.

However, as previously shown in a research publication by Hahne and Aggoun [5], it is an alternative approach to trace rays from the sensor to the object space. In the present paper, a proposition is made to estimate the baseline on the basis of geometrical optics.

\section{RAY TRACING MODEL}

In the following remarks, the plenoptic camera model is simplified to one dimension along the optical axis $z_{U}$ of the main lens. Thus, to apply definitions made hereafter in horizontal and vertical directions equally, the optical system is required to be pointsymmetric meaning that optical parameters of both dimensions are equal in size. Besides, in order to support this requirement, the arrangement of the micro lens array has to be square. The optical planes are denoted as $U$ for the main lens aperture, $s$ for the MLA, whereas each micro lens is indexed by $j$ forming micro images along $u$ indexed by $i$.

As suggested by $\mathrm{Ng}$ et al. [3], the characteristic of the standard plenoptic camera is that the spacing between the MLA and the image plane of the sensor equals the focal length $f_{s}$ of a micro lens. Taking advantage of this constraint and by considering the thin lens equation, it is mathematically demonstrated that rays starting from the sensor converge at an infinite distance from the micro lens [5]. Therefore, light rays can be seen as travelling parallel to each other. Moreover, rays emerging from the same position $u_{i}$ underneath each micro lens have the same slope ratio $m_{i}$ and form a light beam consisting of collimated rays between the micro lens and the main lens. Refering to the underlying theory of collimated light travelling through a lens, light rays are refracted at $U$ and paraxially converge at the focal plane $F_{U}$. The ray tracing model proposed by Hahne et al. [5] is illustrated in Figure 1. For reasons of lucidity, only chief rays are pictured. Additionally, in the drawing it is assumed that the principal planes of the main lens coincide. Nevertheless, a Gaussian lens system is considered in the experimental implementation.

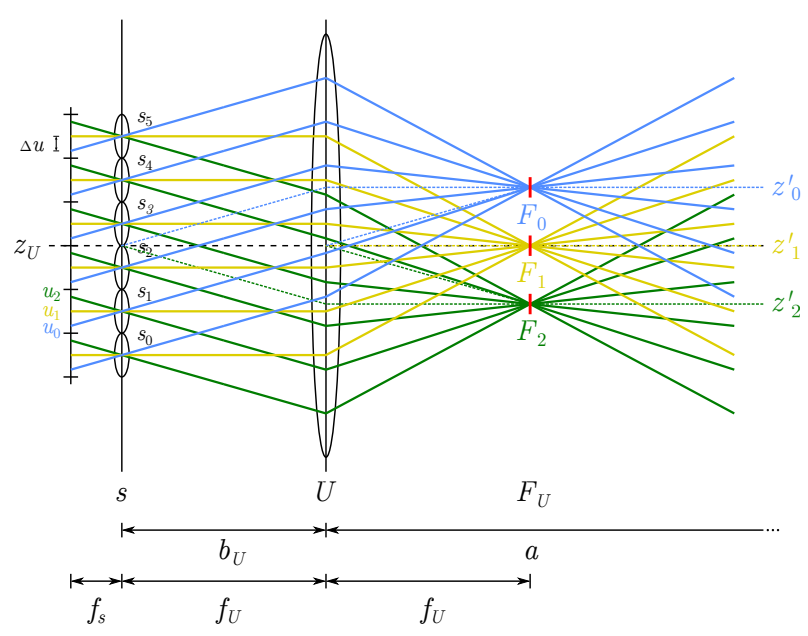

(a)

Figure 1. Virtual camera array through ray tracing

To satisfy the proposed model, elements of the image sensor would have to be of an infinitesimal size which is not the case. However, cells of the image sensor have some spacing $d$ being the pixel size. Assuming the circle of confusion of the point spread function $h$ of the micro lenses to be less or equal the pixel size $(h \leq d)$, compared to a real camera array, the optical center of a virtual camera is rather a line than a point. The length of this line depends on the circle of confusion $h$ or pixel size $d$, respectively. 


\subsection{Paraxial equations}

The slope ratio $m_{i}$ of a chief ray at micro image position $u_{i}$, separated by $\Delta u$ from the micro image center $u_{c}$, is given by

$$
m_{i}=\frac{\Delta u}{f_{s}} .
$$

In order to acquire the position $n$ of a single chief ray at the sensor, the micro lens pitch $p$ has to be taken into account so that

$$
n_{i, j}=s_{j} \times p+\frac{p}{2}+\Delta u
$$

Having calculated the slope $m_{i}$ and its position $n_{i, j}$, a linear equation representing a light ray $m_{i}$ travelling from the image plane through the micro lens to the main lens is formed by

$$
f(z)=m_{i} \times z+n_{i, j}, \quad z \in\left[0, f_{s}+b_{U}\right],
$$

where $b_{U}$ denotes the distance between the main lens $U$ and the MLA $s$. Similarly, the intersection of corresponding chief rays $m_{i}$ at the main lens principal plane $U$, is obtained as

$$
U_{i, j}=m_{i} \times\left(f_{s}+b_{U}\right)+n_{i, j} .
$$

As light rays $m_{i}$ are considered to be collimated in the space between $s$ and $U$, a light beam composed of rays $m_{i}$ can be seen to focus at the main lens focal length $f_{U}$ in object space. In paraxial ray tracing, the focused beam location $F_{i}$ along the focal plane $F_{U}$ depends on the respective slope $m_{i}$ and $f_{U}$ which is given by

$$
F_{i}=m_{i} \times f_{U}
$$

Therefore, chief rays $m_{i}$ are refracted at $U_{i, j}$ in order to converge at $\left(F_{U}, F_{i}\right)$ and diverge subsequently.

\subsection{Virtual cameras}

In previous research [3] it has been stated that collecting all pixels having $u_{i}$ in common and rearranging their illuminance values $E$ consecutively to a single image $E_{u_{i}}^{\prime}$ yields a virtual viewpoint as it would have been captured in a real camera array. Mathematically, a One-Dimensional (1-D) viewpoint image is formed by

$$
E_{u_{i}}^{\prime}\left(s_{j}\right)=E\left(u_{i}, s_{j}\right),
$$

though the given synthesis equation can be applied to two dimensions in order to generate a two-dimensional image. As a consequence, the effective resolution of a virtual camera equals the total number of micro lenses.

Following subsection 2.1, the optical center of a virtual camera providing its viewpoint is approximately at the best focus of converging rays $m_{i}$ sharing the same micro image location $u_{i}$. The optical axis $z_{i}^{\prime}$ of a virtual camera is represented by a viewpoint chief ray being underneath the central micro lens if the number of micro lenses is odd or at an averaged position of the two central micro lenses in case the number is even. Therefore, the axis of a virtual camera perpendicular to $z_{i}^{\prime}$ represents the tilt angle $\Phi_{i}$. Thus, in order to align the tilt angle axis to be perpendicular to $z_{U}, z_{i}^{\prime}$ has to be parallel to the main lens optical axis $\left(z_{i}^{\prime} \| z_{U}\right)$.

Accordingly, this condition is fulfilled when the separation between the main lens and the micro lenses amounts to the focal length of the main lens $\left(f_{U}=b_{U}\right)$ as shown in Figure 1. Geometrically, a tilt angle is obtained by

$$
\Phi_{i}=\arctan \left(\frac{m_{i} \times\left(b_{U}-f_{U}\right)}{f_{U}}\right) .
$$

Different tilt angles of virtual cameras with a varying $b_{U}$ are depicted in Figure 2.

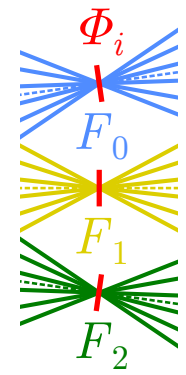

(a) $b_{U}<f_{U}$

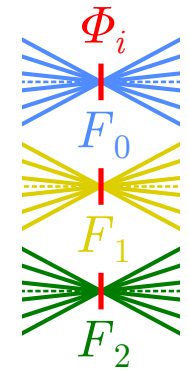

(b) $b_{U}=f_{U}$

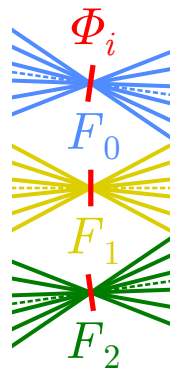

(c) $b_{U}>f_{U}$
Figure 2. Tilt angles $\Phi_{i}$ of the virtual cameras $F_{i}$

\subsection{Baseline estimation}

Using a standard plenoptic setup, the position $\left(F_{U}, F_{i}\right)$ represents the optical center of a virtual camera. Hence, each pixel position within the micro images corresponds to a virtual camera. In an ideal, paraxial lens system, ray tracing can be done by disregarding aberrations. In that case the 1-D positions of two different $F_{i}$ lead to the respective baseline

$$
\Delta B_{i}=\left|F_{i}-F_{c}\right|,
$$

where $F_{c}$ represents the virtual camera position of the central viewpoint with a slope $m_{c}=0$. Figure 3 visualises this principle.

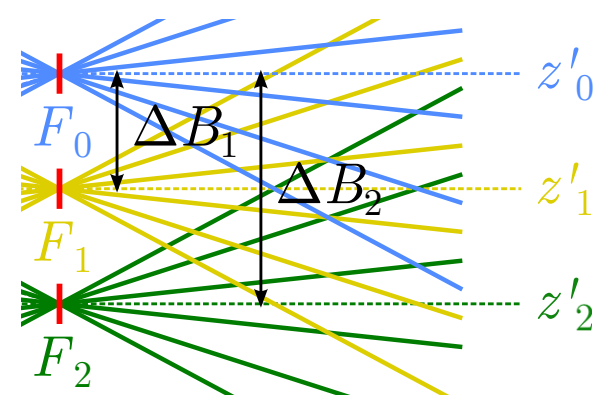

(a)

Figure 3. Baseline $\Delta B_{i}$ of virtual camera setup

This approach can be utilized to estimate the baseline between any virtual cameras whereas $i$ determines the gap. For instance, a gap of $i=1$ means that viewpoints are direct neighbours. Given the requirements of a rectangular micro lens arrangement, the baseline estimation can certainly be applied to horizontal and vertical dimensions equally. 


\section{EXPERIMENTS}

To assess the proposed model, equations elaborated in section 2 have been implemented in Matlab. In order to prove the concept of the baseline estimation, a simulation tool called Zemax has been used for real ray tracing. The optical elements were designed as follows

- micro lens pitch $p=300 \mu \mathrm{m}$

- micro lens focal length $f_{s}=2 \mathrm{~mm}$

- thickness $t_{s}=1.1 \mathrm{~mm}$

- pixel pitch $d=20 \mu \mathrm{m}$

whereas $\Delta u_{i}$ is obtained by

$$
\Delta u_{i}=d \times i
$$

Chromatic aberrations are eliminated by limiting the light spectrum to a wavelength of $\lambda=632.8 \mathrm{~nm}$. The plenoptic camera has been modeled with a Double Gaussian 28 degree field lens provided by Zemax. Whereby, $f_{U}$ amounts to $99.515 \mathrm{~mm}$ and the spacing between principal planes of the objective lens is $t_{U}=-33.184 \mathrm{~mm}$. Screenshots of the model are depicted in Figure 4. Table 1 shows results of the given parameterization.

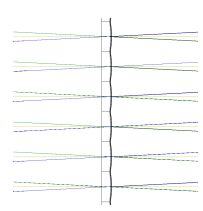

(a) MLA

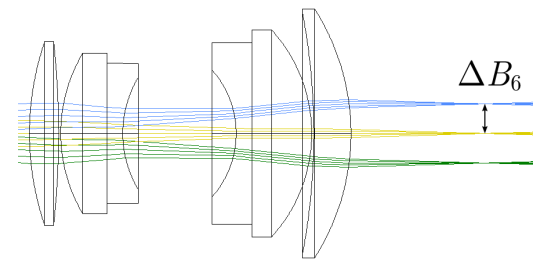

(b) Objective lens
Figure 4. Real ray tracing in Zemax

\begin{tabular}{ccccccc}
\hline Gap & \multicolumn{2}{c}{ Matlab } & \multicolumn{2}{c}{ Zemax } & \multicolumn{2}{c}{ Error } \\
\hline \multirow{2}{*}{$i$} & $\Delta B_{i}$ & $\Phi$ & $\Delta B_{i}$ & $\Phi$ & $\Delta B_{i}$ & $\Phi$ \\
& {$[\mathrm{mm}]$} & {$\left[{ }^{\circ}\right]$} & {$[\mathrm{mm}]$} & {$\left[{ }^{\circ}\right]$} & {$[\%]$} & {$[\%]$} \\
\hline 1 & 0.995 & 0.000 & 0.995 & 0.000 & 0.000 & - \\
\hline 2 & 1.990 & 0.000 & 1.990 & 0.000 & 0.000 & - \\
\hline 3 & 2.985 & 0.000 & 2.985 & 0.000 & 0.000 & - \\
\hline 4 & 3.981 & 0.000 & 3.980 & 0.001 & 0.025 & - \\
\hline 5 & 4.976 & 0.000 & 4.972 & 0.001 & 0.080 & - \\
\hline 6 & 5.971 & 0.000 & 5.963 & 0.002 & 0.134 & - \\
\hline
\end{tabular}

Table 1. Comparison of estimated and simulated $\Delta B_{i}$ where $b_{U}=f_{U}$.

The increasing deviation error is believed to be due to aberrations. As can be seen from Table 2, the main lens focus does not affect the baseline, but rather the tilt angle of virtual cameras.

\begin{tabular}{ccccccc}
\hline Gap & \multicolumn{2}{c}{ Matlab } & \multicolumn{2}{c}{ Zemax } & \multicolumn{2}{c}{ Error } \\
\hline$i$ & $\Delta B_{i}$ & $\Phi$ & $\Delta B_{i}$ & $\Phi$ & $\Delta B_{i}$ & $\Phi$ \\
& {$[\mathbf{m m}]$} & {$\left[{ }^{\circ}\right]$} & {$[\mathbf{m m}]$} & {$\left[^{\circ}\right]$} & {$[\%]$} & {$[\%]$} \\
\hline 1 & 0.995 & 0.115 & 0.995 & 0.115 & 0.000 & 0.000 \\
\hline 2 & 1.990 & 0.230 & 1.990 & 0.230 & 0.000 & 0.000 \\
\hline 3 & 2.985 & 0.346 & 2.985 & 0.346 & 0.000 & 0.000 \\
\hline 4 & 3.981 & 0.461 & 3.978 & 0.462 & 0.075 & 0.217 \\
\hline 5 & 4.976 & 0.576 & 4.971 & 0.578 & 0.101 & 0.347 \\
\hline 6 & 5.971 & 0.691 & 5.962 & 0.694 & 0.151 & 0.434 \\
\hline
\end{tabular}

Table 2. Comparison of estimated and simulated $\Delta B_{i}$ where $b_{U}=f_{U}+$ $20 \mathrm{~mm}$.

Given the suggested ray tracing model, it becomes obvious that baseline estimation results yield to a maximum error of only $0.151 \%$ compared to a real ray tracing simulation tool which, on the contrary, considers spheric and coma aberrations. In relation to a human stereoscopy $(\Delta B=60-70 \mathrm{~mm})$, results of the proposed baseline estimation based on a standard plenoptic camera are significantly smaller. However, the plenoptic camera may be utilised for scenarios where the baseline has to be smaller than the diameter of a typical objective lens. Hence, practical applications of that kind would be in medical imaging, e.g. microscopy or in the field of stop-motion movie production. The presented approach is a novel solution which has advantages over the simulation as it is easy to implement and baseline estimates are computed faster than in a cost-intensive real ray tracing environment like Zemax.

\section{CONCLUSION}

The present research has produced an approach to estimate the baseline of viewpoints obtained by a standard plenoptic camera. Experimental results carried out in Zemax deviate negligibly from the proposed geometric approach. It is a task for further research to align plenoptic image data to be replayed on autostereoscopic monitors by using the proposed approach. For that purpose, it is necessary to design a standard plenoptic camera and a multiview display matching baseline specifications or deploying interpolation techniques to satisfy the baseline of both imaging systems.

\section{REFERENCES}

[1] M. G. Lippmann, "Épreuves réversibles donnant la sensation du relief," Académie Des Sciences, pp. 446-451, March 1908.

[2] E. H. Adelson and J. Y. A. Wang, "Single lens stereo with a plenoptic camera," IEEE TRANSACTIONS ON PATTERN ANALYSIS AND MACHINE INTELLIGENCE, vol. 14, no. 2, pp. 99-106, February 1992.

[3] R. Ng, M. Levoy, M. Brèdif, G. Duval, M. Horowitz, and P. Hanrahan, "Light field photography with a hand-held plenoptic camera," Tech. Rep. CTSR 2005-02, Stanford University, 2005.

[4] T. Georgiev, A. Lumsdaine, and S. Goma, "Plenoptic principal planes," in Imaging and Applied Optics. 2011, p. JTuD3, Optical Society of America.

[5] C. Hahne and A. Aggoun, "Embedded fir filter design for realtime refocusing using a standard plenoptic video camera," in Digital Photography X. 2014, SPIE. 